\title{
Biomarcadores sanguíneos de caprinos Saanen com diferentes faixas etárias*
}

\author{
Blood biomarkers of saanen goats with different ages \\ Elizabeth Regina Rodrigues Silva, ${ }^{* *}$ Monica Miranda Hunka, ${ }^{* *}$ Maria Presciliana de Brito Ferreira, ${ }^{* *}$ \\ Telga Lucena Alves Craveiro Almeida, ${ }^{* *}$ Simone Gutman Vaz, ${ }^{* *}$ Stephânia Katurchi Mendes Mélo, ${ }^{* *}$ \\ Helena Emília Cavalcanti da Costa Cordeiro Manso, ${ }^{* *}$ Hélio Cordeiro Manso Filho**
}

\begin{abstract}
Resumo
Objetivou-se estabelecer o perfil aminoacídico, bioquímico e hematológico de caprinos sadios da raça Saanen de diferentes faixas etárias criados em regime intensivo no Nordeste do Brasil. A partir de amostras de sangue de cada animal foi realizada a análise de aminoácidos, perfil hematológico e bioquímico. Os dados foram submetidos a ANOVA e ao Teste de Tukey $(P<0,05)$. A concentração de glutamato [GLU] e a de glutamina e glutamato [GLN+GLU] apresentaram variações $(P<0,05)$ enquanto que a [GLN] não variou $(P>0,05)$. A [GLU] e de [GLN+GLU] foram $91 \%$ e $43 \%$ superiores nos animais do grupo cria quando comparados com os demais. Ocorreram também valores significativos nas concentrações de proteínas plasmáticas totais [PPT], ureia [URE], creatinina [CREAT], ácido úrico [AcU], alanina aminotransferase [ALT], creatina quinase [CK], glicose [GLIC], triglicérides [TRIG] e colesterol total [COLES-T] $(P<0,05)$, diferentemente das albumina [ALB] e aspartato aminotransferase [AST]. Nos índices hematológicos houve diferenças para volume corpuscular médio (VCM), concentração de hemoglobina corpuscular média (CHCM), coeficiente de variação de amplitude de distribuição dos eritrócitos (RDW-CV) $(P<0,05)$, mas não ocorreram variações significativas de resultados nas células brancas do sangue [CGB], hemácias [HEM], hemoglobina [Hb] e na percentagem do hematócrito $(P>0,05)$. Destaca-se que este conhecimento possibilite melhor entendimento dos processos metabólicos nos animais hígidos e enfermos, levando em consideração as condições alimentar e do manejo da região. Contribuindo assim para aumentar a produtividade do rebanho na região tropical dada a grande importância da caprinocultura no Nordeste do Brasil.
\end{abstract}

Palavras-chave: aminoácido, bioquímica, caprino, hematologia.

\section{Abstract}

The aim of this study was to establish biochemical, hematological and amino acid profiles from healthy Saanen goats, from different ages bred on intensive system, in Northeastern Brazil. It was used blood Samples to analyse these parameters. Data was submitted to ANOVA and Tukey test $(P<0.05)$. The glutamate [GLU] and glutamine plus glutamate [GLN+GLU] showed differences $(P<0.05)$, while [GLN] didn't $(P>0.05)$. The [GLU] and [GLN+GLU] was $91 \%$ and $43 \%$ higher on post-weaning group. Also it was seen significance on total protein plasmatic [TPP], urea [URE], creatinine [CREAT], uric acid [UAC], alanine aminotransferase [ALT], creatine kinase [CK], glucose [GLU], total triglyceride [TG] and total cholesterol [CHOL]. Unlike albumin [ALB] and aspartate aminotransferase [AST]. Haematological indices were no differences of mean corpuscular volume (MCV), mean corpuscular hemoglobin concentration (MCHC), coefficient of variation of amplitude red cell distribution (RDW-CV) $(P<0.05)$, but no significant changes in results in white blood cell [WBC], [HEM] red blood cells, hemoglobin $[\mathrm{Hb}]$ and the percentage of hematocrit $(P>$ 0.05). Metabolic processes on healthy and sick animals, should be better understood by this study, considering nutritional and environmental condition in this region. This contribution may increase herd productivity in this region by the great importance of goat breeding in northeastern Brazil.

Keywords: amino acid, biochemistry, goat, hematology.

\section{Introdução}

Nos últimos anos, tem-se observado o crescimento da caprinocultura no país, em parte devido às vantagens que esse tipo de criação apresenta em pequenas áreas, como ao menor consumo de alimentos, facilidade de manejo e uma boa diversidade de produção (Bezerra et al., 2008).
Os caprinos são criados em diferentes partes do mundo e informações a respeito dos biomarcadores hematológicos e bioquímicos podem ser encontrados para diferentes raças e sistemas de manejo. Entretanto, ainda são reduzidas as informações no que se refere ao metabolismo da glutamina e do glutamato e as suas relações com outros parâmetros metabólicos e sanguíneos sob condições tropicais (Greenwood

\footnotetext{
*Recebido em 24 de março de 2015 e aceito em 23 de abril de 2017.

**Laboratório de Biotecnologia Molecular Aplicada à Produção Animal (BIOPA), Departamento de Zootecnia, Universidade Federal Rural de Pernambuco (UFRPE).

Autora para correspondência: bethrrs@yahoo.com.br
} 
e McBride, 2010; Zavarize et al., 2010; Caroprese et al., 2012; Oliveira et al., 2012).

Desta forma, objetivou-se estabelecer o perfil aminoacídico, bioquímico e hematológico de caprinos sadios da raça Saanen de diferentes faixas etárias criadas em regime intensivo. Esperase que este conhecimento possibilite melhor entendimento dos processos metabólicos nos animais sadios e enfermos contribuindo para sua produtividade.

\section{Material e métodos}

O experimento foi conduzido no setor de caprinos do Departamento de Zootecnia da Universidade Federal Rural de Pernambuco (UFRPE), campus Recife. Foram utilizados 24 caprinos da raça Saanem escolhidos aleatoriamente, de ambos os sexos, criados intensivamente e separados por lotes. Todos eram mantidos em regime nutricional seguindo as indicações do NRC (National Research Council, 2007) para a espécie e por categoria, através do fornecimento de dieta à base de feno de Tifton (Cynodon dactylon, cv. Tifton), concentrado à base de milho, soja e farelo de trigo. O fornecimento de água e sal mineralizado era ad libitum. Este experimento foi submetido e aprovado pela Comissão de Ética no Uso de Animais em Pesquisa CEUA/ UFRPE, sob o processo n. 23082.016781/2008.

Os animais foram divididos em quatro grupos conforme a faixa etária e estádio produtivo: cria (10 $\pm 2,37$ dias), recria $(90 \pm 6,45$ dias), gestante ( $3 \pm 0,10$ anos) e lactante ( $3 \pm 0,08$ anos). Todos os animais eram vacinados e vermifugados regularmente seguindo as práticas do setor.

Foram colhidas amostras sanguíneas por meio de venopunção da jugular externa, utilizando-se o sistema de coleta a vácuo, em tubos previamente resfriados. Para as análises hematológicas e de aminoácidos: glutamina (GLN) e glutamato (GLU) utilizaramse tubos com heparina (Vacuette ${ }^{\circledR}$, Greiner Bio-One, Brasil). Para o estudo bioquímico tubos sem anticoagulante foram empregados. As amostras foram refrigeradas e imediatamente enviadas ao laboratório para realizar as análises.

A contagem de células sanguíneas foi realizada utilizando-se contador de células automatizado (Sysmex Poch - 100iV Diff, Roche Diagnóstica, Brasil), fornecendo os seguintes parâmetros: CGV (células vermelhas do sangue), HB (hemoglobina), HT (hematócrito), VCM (volume corpuscular médio), $\mathrm{CHCM}$ (concentração de hemoglobina corpuscular média), CGB (total de células brancas do sangue) e RDW - CV (coeficiente de variação de amplitude de distribuição dos eritrócitos). As análises bioquímicas foram realizadas utilizando kits comerciais (Doles, Brasil) com o uso de um analisador semi-automático (Doles D-250, Doles, Brasil), fornecendo os seguintes parâmetros: ureia (URE), creatinina (CREAT), alanino aminotransferase $(A L T)$, aspartato aminotransferase (AST), albumina (ALB), ácido úrico $(A U)$, glicose (GLI), triglicérides (TRIG), colesterol total (COL) e creatina quinase (CK). A dosagem das PPT (proteínas plasmáticas totais) foi realizada através da refratometria manual.
As concentrações de glutamato (GLU) e glutamina (GLN) foram analisadas pelo método de detecção enzimática com leitura em espectrofotometria a 340nm (Manso Filho et al., 2008).

Os resultados foram submetidos à análise da variância (ANOVA), o teste de Tukey foi utilizado como teste post hoc, utilizando-se o programa computacional SigmaStat 3.0 para Windows. Em ambos os casos o valor de $P$ foi estabelecido em $5 \%$. Os resultados estão expressos na forma de média +/- erro padrão médio.

\section{Resultados e discussão}

Demonstrou-se que a [GLU] e a [GLN+GLU] apresentaram variações significativas enquanto a [GLN] não variou $(P>0,05)$ (Tabela 1). A [GLU] e de [GLN+GLU] foram $91 \%$ e $43 \%$ superiores nos animais do grupo cria quando comparados com os demais grupos.

Tabela 1: Concentração sanguínea do glutamato e da glutamina de caprinos da raça Saanen em diferentes categorias.

\begin{tabular}{lcccc}
\hline \multirow{2}{*}{ Aminoácido } & \multicolumn{4}{c}{ Categoria Animal } \\
\cline { 2 - 5 } & Cria & Recria & Gestante & Lactante \\
\hline Glutamato & $0,44 \pm 0,06^{\mathrm{A}}$ & $0,22 \pm 0,03^{\mathrm{B}}$ & $0,25 \pm 0,03^{\mathrm{B}}$ & $0,25 \pm 0,03^{\mathrm{B}}$ \\
Glutamina & $0,45 \pm 0,08$ & $0,39 \pm 0,06$ & $0,39 \pm 0,04$ & $0,38 \pm 0,05$ \\
GLU + GLN & $0,90 \pm 0,08^{\mathrm{A}}$ & $0,62 \pm 0,05^{\mathrm{B}}$ & $0,64 \pm 0,05^{\mathrm{B}}$ & $0,63 \pm 0,06^{\mathrm{B}}$ \\
\hline
\end{tabular}

esma linha indicam $P<0,05$ pelo teste Tukey.

Com exceção da [ALB] e [AST], também foram observadas variações nas concentrações séricas de [PPT], [URE], [CREAT], [AcU], [ALT], [CK], [GLIC], [TRIG] e [COLES-T] $(\mathrm{P}<0,05)$ (Tabela 2).

Em relação aos índices hematológicos, houve variação significativa para VCM, CHCM, RDW-CV $(P<0,05)$, mas não ocorreram variações na [CGB], [HEM], [Hb] e na percentagem do hematócrito $(P>0,05)$ (Tabela 3).

Além de ser significativa fonte energética para os enterócitos e as células do sistema imune, a glutamina (GLN) é precursora de nucleotídeos, moléculas importantes no desenvolvimento e reparo de células imunes e intestinais (Bergstrom e Krebs, 2004).

A [GLN] não apresentou variação nas diferentes faixas etárias, embora animais na fase de cria apresentassem maiores concentrações de GLN e também na fase de recria onde há mudança nos hábitos alimentares após o desmame, e com isso novos desafios para as células intestinais, além destes animais não serem capazes de sintetizar GLN em quantidades suficientes. Nos animais lactentes e recém-desmamados, há extensa degradação no enterócito de Glutamina e Leucina (Rhoads e Wu, 2009).

Li et al. (2009) alegam o papel funcional da glutamina como aminoácido funcional regulador das vias metabólicas durante o desenvolvimento fetal e neonatal. $E$ ainda participa na composição aminoacídica do leite materno e crescimento de potros (Matsui et al., 2005). 
Tabela 2: Média do perfil bioquímico de caprinos da raça Saanen em diferentes categorias

\begin{tabular}{lcccc}
\hline \multirow{2}{*}{ Biomarcador } & \multicolumn{4}{c}{ Fases de vida } \\
\cline { 2 - 5 } & Cria & Recria & Gestante & Lactante \\
\hline PPT (g/dL) & $5,45 \pm 0,24^{\mathrm{C}}$ & $6,73 \pm 0,19^{\mathrm{AB}}$ & $6,13 \pm 0,24^{\mathrm{ABC}}$ & $6,75 \pm 0,32^{\mathrm{A}}$ \\
\hline Albumina (g/dL) & $2,52 \pm 0,11$ & $2,74 \pm 0,16$ & $2,35 \pm 0,24$ & $2,25 \pm 0,25$ \\
\hline Ureia (mg/dL) & $21,59 \pm 3,93^{\mathrm{C}}$ & $39,26 \pm 2,12^{\mathrm{AB}}$ & $33,50 \pm 2,24^{\mathrm{B}}$ & $46,33 \pm 1,44^{\mathrm{A}}$ \\
\hline Creatinina (mg/dL) & $0,81 \pm 0,05^{\mathrm{B}}$ & $0,88 \pm 0,03^{\mathrm{B}}$ & $1,00 \pm 0,05^{\mathrm{A}}$ & $0,82 \pm 0,02^{\mathrm{B}}$ \\
\hline Ácido Úrico (mg/dL) & $2,01 \pm 0,03^{\mathrm{A}}$ & $1,92 \pm 0,01^{\mathrm{B}}$ & $1,97 \pm 0,03^{\mathrm{AB}}$ & $1,9 \pm 0,01^{\mathrm{B}}$ \\
\hline ALT (U/L) & $4,39 \pm 1,18^{\mathrm{B}}$ & $12,62 \pm 2,37^{\mathrm{A}}$ & $9,60 \pm 1,18^{\mathrm{A}}$ & $11,67 \pm 1,94^{\mathrm{A}}$ \\
\hline AST (U/L) & $51,93 \pm 3,62$ & $109,31 \pm 37,70$ & $72,75 \pm 6,81$ & $72,39 \pm 5,77$ \\
\hline CK (U/L) & $140,63 \pm 16,75^{\mathrm{AB}}$ & $185,06 \pm 38,69^{\mathrm{A}}$ & $81,69 \pm 10,30^{\mathrm{B}}$ & $93,06 \pm 12,37^{\mathrm{B}}$ \\
\hline Glicose (mg/dL) & $105,69 \pm 8,07^{\mathrm{A}}$ & $91,17 \pm 15,32^{\mathrm{AB}}$ & $56,54 \pm 8,04^{\mathrm{C}}$ & $69,89 \pm 3,10^{\mathrm{BC}}$ \\
\hline Triglicerídeos (mg/dL) & $60,65 \pm 9,33^{\mathrm{A}}$ & $44,09 \pm 4,07^{\mathrm{AB}}$ & $36,01 \pm 3,45^{\mathrm{B}}$ & $36,17 \pm 5,13^{\mathrm{B}}$ \\
\hline Colesterol (mg/dL) & $153,47 \pm 11,70^{\mathrm{A}}$ & $98,88 \pm 6,57^{\mathrm{B}}$ & $85,72 \pm 5,72^{\mathrm{B}}$ & $100,14 \pm 7,00^{\mathrm{B}}$ \\
\hline
\end{tabular}

*Diferentes letras na mesma linha significam $\mathrm{P}<0,05$, pelo teste Tukey.

Tabela 3: Variação nos parâmetros hematológicos de caprinos da raça Saanen em diferentes categorias

\begin{tabular}{|c|c|c|c|c|}
\hline \multirow{2}{*}{ Variáveis } & \multicolumn{4}{|c|}{ Categoria Animal } \\
\hline & Cria & Recria & Gestante & Lactante \\
\hline CGB $\left(x 10^{3} / \mu \mathrm{L}\right)$ & $11,9 \pm 1,24^{\mathrm{A}}$ & $10,28 \pm 0,48^{A}$ & $10,95 \pm 0,70^{A}$ & $10,3 \pm 0,90^{A}$ \\
\hline $\operatorname{HEM}\left(x 10^{6} / \mu \mathrm{L}\right)$ & $10,91 \pm 0,66^{A}$ & $11,43 \pm 0,50^{A}$ & $10,68 \pm 0,40^{A}$ & $10,09 \pm 0,86^{A}$ \\
\hline $\mathrm{HGB}(\mathrm{g} / \mathrm{dL})$ & $6,72 \pm 0,43^{A}$ & $6,43 \pm 0,31^{A}$ & $6,18 \pm 0,32^{\mathrm{A}}$ & $5,85 \pm 0,46^{A}$ \\
\hline $\mathrm{HCT}(\%)$ & $26,23 \pm 0,74^{A}$ & $22,8 \pm 0,29^{A}$ & $23,46 \pm 0,37^{A}$ & $21,68 \pm 0,45^{A}$ \\
\hline VCM (fL) & $23,95 \pm 0,74^{\mathrm{A}}$ & $20,01 \pm 0,29^{B}$ & $21,93 \pm 0,37^{B}$ & $21,61 \pm 0,45^{\mathrm{B}}$ \\
\hline $\mathrm{CHCM}(\mathrm{g} / \mathrm{dL})$ & $25,75 \pm 0,52^{B}$ & $28,3 \pm 1,03^{A}$ & $26,31 \pm 0,35^{\mathrm{AB} B}$ & $26,96 \pm 0,15^{\mathrm{AB} A B}$ \\
\hline RDW-CV (\%) & $39,01 \pm 2,12^{\mathrm{A}}$ & $29,38 \pm 0,78^{B}$ & $27,11 \pm 0,40^{\mathrm{B}}$ & $26,2 \pm 0,33^{\mathrm{B}}$ \\
\hline
\end{tabular}

${ }^{*}$ Diferentes letras na mesma linha indicam $\mathrm{P}<0,05$ pelo teste Tukey.

Em vacas, Doepel et al. (2006) afirmaram que demandas metabólicas de GLN podem ser notadamente elevadas no início da lactação, quando a demanda para a síntese de proteína no leite é acompanhada por um grande aumento no intestino e na glândula mamária, como também um grande consumo energético de todo o corpo.

Vale ressaltar que as crias receberam a GLN na nutrição através do leite das lactantes. Embora a concentração de GLN nesta fase não tenha sofrido alteração, devido à produção de glutamina também acontecer em parte na glândula mamária evoluindo ao longo da lactação. As amostras das crias foram coletadas com os animais muito jovens, motivo também de não ter havido diferenças. A lactação é um estado fisiológico complexo e bem elaborado, disponibilizando proteína no leite a partir do catabolismo das reservas proteicas corporal, sobretudo do sistema músculoesquelético (Clowes et al., 2005; Matsui et al., 2005). A suplementação com GLN diminui a utilização de GLN plasmática em até 40\% (D' Paula, 2013). Embora não tenham mostrado diferenças, permitiriam maior aporte de GLN plasmáticas para as células de defesa destes animais.

O glutamato, especialmente o derivado da dieta, pode facilmente substituir a glutamina em diversos dos seus papéis metabólicos, incluindo a geração de energia e a síntese de aminoácidos. A [GLU] e [GLU+GLN] mostrou-se mais elevada na fase de cria $(P<0,05)$, sendo sua concentração semelhante para as demais fases do experimento. Esta diferença pode ser justificada pela deficiência da glutamina sintetase de animais nesta fase da vida, mantendo elevação deste índice que serve como substrato para síntese de GLN através desta enzima.

O estudo das proteínas plasmáticas pode indicar modificações nos níveis nutricionais ou afecções em tecidos ligados ao metabolismo desses biomarcadores. Vários tecidos estão envolvidos no metabolismo de proteínas plasmáticas, e o fígado é o principal (Barioni et al., 2001). $\mathrm{A}[\mathrm{PPT}]$ apresentou menores resultados na fase de cria, $(P<0,05)$, enquanto a $[A L B]$ não se alterou. Valor semelhante a outros estudos (Brito, 2008) com valores baixos em animais da mesma raça e idade $(5,15$ $\mathrm{g} / \mathrm{dL}$ ). Provavelmente, as crias da raça Saanen são menos adaptadas a absorção de imunoglobulinas alcançadas através da ingestão do colostro.

As provas de função renal são ilustradas pelas concentrações séricas médias de ureia e creatinina. Ambos apresentaram diferenças entre as fases de vida, com [URE] mais elevada na fase de lactação e [CREAT] mais marcante na fase de gestação. A concentração sanguínea de ureia mantém relação direta com o aporte de proteínas da ração, bem como da relação energia:proteína. Desta forma, tal variação deve-se à diferenciação de alimentação que estes animais recebem em diferentes fases da vida. Todos os valores encontrados estão dentro dos padrões de normalidade (Kaneko et al., 2008). A [CREAT] menor na fase de cria pode ser justificada pela menor massa muscular apresentada nesta fase da vida. 
Segundo Yu et al. (2002), o valor do ácido úrico pode ser afetado pelas fontes de proteína dietética e energia, pelo consumo de matéria seca, proteína, peso vivo, aditivos alimentares e pela espécie. Os teores de ácido úrico apresentaram diferenças marcantes, quando se comparou entre os grupos, sobretudo nas categorias cria e recria. Possivelmente esta alteração se dá pela diferenciação na alimentação que esses animais passam da fase de cria, onde se alimentam apenas do leite e na fase de recria onde é introduzido o concentrado.

As enzimas ALT e AST fornecem informações sobre a função hepática e AST e CK são as mais utilizadas para avaliação do sistema muscular (Harris et al., 1998). A [ALT] apresentou-se menor na fase de cria, o que possivelmente está relacionado com a imaturidade hepática, enquanto a [AST] não variou nas diferentes fases do experimento. Há divergências na literatura quanto às variações de AST em função da faixa etária. Behera et al. (1993), encontraram variação significativa em relação à idade dos animais. Em relação à [CK], os maiores resultados estão na fase de cria e recria. É importante considerar que a CK é uma enzima citoplasmática sujeita a liberação rápida na circulação, como resultado de pequenas alterações celulares.

No que se refere à caracterização do estado energético dos ruminantes avaliou-se a [GLIC], [COLEST] e [TRIG], pois constituem determinações realizadas na rotina laboratorial. A [GLIC] apresentou valores mais elevados na fase de cria $(P<0,05)$, assim como os valores de COLEST e TRIG. Souza (1997) observou que após o desmame os teores de glicose sofrem redução significativa em função da modificação da alimentação e do metabolismo energético. Por esta razão, devese considerar a idade dos animais quando se analisa a glicemia. A principal fonte energética do leite está disponível na forma de lipídios e como consequência ocorre elevação dos lipídios plasmáticos que reflete no perfil bioquímico, principalmente pelos teores séricos de colesterol. Estudos revelam a influência dos fatores etários nas concentrações séricas de triglicérides, colesterol, ácidos graxos livres não esterificados (NEFA) e glicose sobretudo nos primeiros seis meses de vida (Pogliani e Birgel, 2007).

O valor de glóbulos vermelhos é uma prova de condição de saúde do animal. A contagem de hemácias, hemoglobina e hematócrito não variaram nas diferentes fases experimentais $(P>0,05)$, demonstrando pouca variabilidade entre animais que estão sob as mesmas condições de manejo. Em relação aos índices hematimétricos, o VCM é mais comumente utilizado, porém, atualmente o RDW-CV vem sendo utilizado, cujo valor reflete, de forma mais sensível, o grau de heterogeneidade entre as hemácias por meio de uma análise quantitativa (Balarin et al., 2006). Observou-se maior valor de RDW-CV no grupo cria, assim como os valores de VCM, demonstrando que os animais mais novos possuem grande quantidade de células jovens. Gama et al. (2007) verificaram a ocorrência de reticulócitos da primeira até a sexta semana de vida, o que pode explicar facilmente a elevação do RDW-CV e VCM nestes animais. Já para o índice $\mathrm{CHCM}$ o grupo cria apresentou valores menores. A contagem de leucócitos não apresentou diferenças entre as fases experimentais.

\section{Conclusão}

Por meio destes resultados conclui-se que alguns biomarcadores variam de acordo com a categoria estudada (GLU, GLN+ GLU, PPT, URE, CREAT, AcU, ALT, CK, GLIC, TRIG, COLEST-T, VCM, CHCM, RDW-CV). Esses animais passam por adaptações decorridas na mudança de alimentação, clima e manejo. Deve-se continuar com pesquisas relacionadas com esses índices, uma vez que pode ainda haver variabilidade em relação ao gênero e sistemas de criação.

\section{Agradecimentos}

Agradecemos o apoio da CAPES e CNPq pela concessão das bolsas de pós-graduação, à Ajinomoto Biolatina pelo apoio ao Laboratório BIOPA/UFRPE e ao Setor de Caprinos UFRPE/DZ.

\section{Referências}

BALARIN, M.R.S. et al. Valores da amplitude de distribuição do tamanho dos eritrócitos (RDW) em equinos puro sangue inglês (PSI) submetidos a exercícios em diferentes intensidades. Brazilian Journal of Veterinary Research and Animal Science,v. 43, n. 5,p. 637-641, 2006.

BARIONI, G. et al. Valores séricos de cálcio, fósforo, sódio, potássio e proteínas totais em caprinos fêmeas da raça parda alpina. Ciência Rural, v. 31, n. 3, p. 435-438, maio/jun. 2001.

BEZERRA, L.R. et al. Perfil hematológico de cabras clinicamente sadias criadas no cariri paraibano. Ciência e Agrotecnologia, v. 32, n. 3, p. 955-960, maio/jun. 2008.

BEHERA, P.C. et al. Serum enzyme activity in different age groups of male and female Black Bengal goats. Indian Veterinary Journal, v. 70, n. 11, p.1042-1045, 1993.

BERGSTROM, J.; KREBS, H.A. Glutamine: metabolism and application in nutrition support. Asia Pacific Journal Clinical Nutrition, v.13, p. 25-31, 2004.
BRITO, R.L. Níveis de proteínas totais, albuminas, globulinas e gamaglobulinas no soro de crias caprinas das raças moxotó e sanen criadas no semiárido nordestino. In: CONGRESSO NORDESTINO DE PRODUÇÃO ANIMAL, 5., 2008, Aracaju, SE. Anais... Aracaju: Universidade Federal de Sergipe, 2008. v. 5. p.1-3.

CAROPRESE, M. et al. An immune response and milk production of dairy cows fed graded levels of rumen-protected glutamine. Research in Veterinary Science. v. 93, n. 1, p. 202-209, ago. 2012.

CLOWES, E.J. et al. Skeletal muscle protein mobilization during the progression of lactation. American Journal Physiology Endocrinology Metabolism, v. 288, n. 1, p. 564-572, mar. 2005.

D'PAULA, J.T. Concentração da glutamina e composição do leite em vacas Holandesas durante a lactação. 2013. 52 f. Dissertação (Mestrado em Ciências Veterinárias) - Curso de Pós-graduação em Ciências Veterinárias, Universidade Federal Rural de Pernambuco. Recife, 2013. 
DOEPEL, L. et al. Effect of post-ruminal glutamine supplementation on immune response and milk production in dairy cows. Journal Dairy Science, v. 89, n. 8, p. 3107-3121, ago. 2006.

GAMA, S.M.S. et al. Dinâmica do eritrograma de cordeiros, resultantes do cruzamento entre animais de raças nativas criadas no Nordeste e a raça Dorper, desde o nascimento até os seis meses de idade. Revista Brasileira de Saúde e Produção Animal, v. 8, n. 1, p. 11-23, 2007.

GREENWOOD, S.L.; McBRIDE, B.W. Development and characterization of the ruminant model of metabolic acidosis and its effects on protein turnover and amino acid status. In: PROCEEDINGS OF THE $4^{\text {th }}$ AUSTRALASIAN DAIRY SCIENCE SYMPOSIUM, 2010.

HARRIS, P.A. et al. Plasma aspartate aminotransferase and creatine kinase activities in thoroughbred racehorses in relation to age, sex, exercise and training. The Veterinary Journal, v. 155, n. 3, p. 295-304, maio 1998.

KANEKO, J.J. et al. Clinical biochemistry of domestic animals. San Diego: Academic Press, 2008, 6 v.

LI, P. et al. Lactating porcine mammary tissue catabolizes branched-chain amino acids for glutamine and aspartate synthesis. The Journal of Nutrition, v. 139, n. 8, p.1502-1509, ago. 2009.

MANSO FILHO, H.C. et al. Distribution of glutamine synthetase and an inverse relationship between glutamine synthetase expression and intramuscular glutamine concentration in the horse. Comparative Biochemistry and Physiology, v. 150, n. 3, p. 326-330, jul. 2008.
MATSUI, A. et al. Effects of acute changes in the energy and protein intake levels over the short-term on the maternal milk amino acid concentrations in lactating mares. Asian -Australian Journal Animal Science, v. 18, n. 6, p. 855-860, 2005.

NATIONAL RESEARCH COUNCIL. Nutrient Requirements of Small Ruminants: Sheep, Goats, Cervids, and New World Camelids. Washington, DC: The National Academies Press, 2007.

OLIVEIRA, M.G.C. et al. Aspectos hematológicos de caprinos (Capra hircus) da raça Canindé criados no Rio Grande do Norte. Revista Pesquisa Veterinária Brasileira, v. 32, n. 1, p. 4-8, dez. 2012.

POGLIANI, F.C.; BIRGEL, J.E.H. Valores de referência do lipidograma de bovinos da raça holandesa, criados no Estado de São Paulo. Brazilian Journal of Veterinary Research and Animal Science, v. 44, p. 373-383, 2007.

RHOADS, J.M.; WU, G. Glutamine, arginine and leucine signaling in the intestine. Amino Acids, v. 37, p.111-122, 2009.

SOUZA, R.P. Perfil bioquímico sérico de bovinos das raças Gir, Holandesa e Girolanda, criados no Estado de São Paulo influência de fatores de variabilidade etários e sexuais. 1997. 168f. Tese (Doutorado) - Faculdade de Medicina Veterinária e Zootecnia, Universidade de São Paulo.

YU, P. et al. Purine derivative excretion and ruminal microbial yield in growing lambs fed raw and dry roasted legume seeds as protein supplements. Animal Feed Science and Technology, v. 95, n. 1-2, p. 33-48, jan. 2002.

ZAVARIZE, K.C. et al. Utilização de glutamina na nutrição de monogástricos. Revista Portuguesa de Ciências Veterinárias, v. 109, n. 573-576, p. 5-10, jan/dez 2010. 